\title{
Exploring the dark matter inelastic frontier with 79.6 days of PandaX-II data
}

\author{
Xun Chen, ${ }^{1, *}$ Abdusalam Abdukerim, ${ }^{2}, \dagger$ Wei Chen, ${ }^{1}$ Yunhua Chen,${ }^{3}$ Xiangyi Cui, ${ }^{1}$ Deqing Fang, ${ }^{4}$ \\ Changbo Fu, ${ }^{1}$ Karl Giboni, ${ }^{1}$ Franco Giuliani, ${ }^{1}$ Xuyuan Guo, ${ }^{3}$ Zhifan Guo, ${ }^{5}$ Ke Han, ${ }^{1}$ Shengming He, ${ }^{3}$ \\ Xingtao Huang, ${ }^{6}$ Xiangdong Ji, ${ }^{7,1}$, Yonglin $\mathrm{Ju},{ }^{5}$ Shaoli Li, ${ }^{1}$ Heng Lin,${ }^{1}$ Huaxuan Liu, ${ }^{5}$ Jianglai Liu,,${ }^{1,7}$ \\ Yugang Ma, ${ }^{4}$ Yajun Mao, ${ }^{8}$ Jinhua Ning, ${ }^{3}$ Xiangxiang Ren, ${ }^{1}$ Fang Shi, ${ }^{1}$ Andi Tan, ${ }^{9}$ Cheng Wang, ${ }^{5}$ \\ Hongwei Wang, ${ }^{4}$ Meng Wang, ${ }^{6}$ Qiuhong Wang, ${ }^{4}$ Siguang Wang, ${ }^{8}$ Xiuli Wang, ${ }^{5}$ Xuming Wang, ${ }^{1}$ Qinyu \\ $\mathrm{Wu},{ }^{1}$ Shiyong Wu,${ }^{3}$ Mengjiao Xiao, $,{ }^{9}, 10$ Pengwei Xie, ${ }^{1}$ Binbin Yan, ${ }^{6}$ Yong Yang, ${ }^{1}$ Jianfeng Yue, ${ }^{3}$ Dan \\ Zhang, ${ }^{1}$ Hongguang Zhang, ${ }^{1}$ Tao Zhang, ${ }^{1}$ Li Zhao, ${ }^{1}$ Jifang Zhou, ${ }^{3}$ Ning Zhou, ${ }^{1}$ and Xiaopeng Zhou ${ }^{8}$ \\ (PandaX-II Collaboration) \\ ${ }^{1}$ INPAC and School of Physics and Astronomy, Shanghai Jiao Tong University, \\ Shanghai Laboratory for Particle Physics and Cosmology, Shanghai 200240, China \\ ${ }^{2}$ School of Physics and Technology, Xinjiang University, Ürümqi 830046, China \\ ${ }^{3}$ Yalong River Hydropower Development Company, \\ Ltd., 288 Shuanglin Road, Chengdu 610051, China \\ ${ }^{4}$ Shanghai Institute of Applied Physics, Chinese Academy of Sciences, 201800, Shanghai, China \\ ${ }^{5}$ School of Mechanical Engineering, Shanghai Jiao Tong University, Shanghai 200240, China \\ ${ }^{6}$ School of Physics and Key Laboratory of Particle Physics and Particle \\ Irradiation (MOE), Shandong University, Jinan 250100, China \\ ${ }^{7}$ T.D. Lee Institute, Shanghai, China \\ ${ }^{8}$ School of Physics, Peking University, Beijing 100871, China \\ ${ }^{9}$ Department of Physics, University of Maryland, College Park, Maryland 20\%42, USA \\ ${ }^{10}$ Center of High Energy Physics, Peking University, Beijing 100871, China
}

(Dated: October 4, 2017)

\begin{abstract}
We report here the results of searching for inelastic scattering of dark matter (initial and final state dark matter particles differ by a small mass splitting) with nucleon with the first 79.6-day of PandaX-II data (Run 9). We set the upper limits for the spin independent WIMP-nucleon scattering cross section up to a mass splitting of $300 \mathrm{keV} / \mathrm{c}^{2}$ at two benchmark dark matter masses of 1 and $10 \mathrm{TeV} / \mathrm{c}^{2}$.
\end{abstract}

After more than 30 years of experimental effort, Weakly Interactive Massive Particles (WIMPs) remain hidden in most recent dark matter direct detection experiments (see Ref. [1] and [2] for a recent review). One possible explanation for the null results in these experiments is that the elastic scattering between the WIMPs and nucleon is heavily suppressed. In some theoretical scenarios [3-5], inelastic scattering, in which the dark matter particle converts to an excited state particle with a small mass splitting between the two, becomes dominant. This could explain the observed annual modulation in the DAMA/LIBRA data [6], but the annual modulation in signal was incompatible with the results from several other different experiments [7, 8]. In a recent study [9], the inelastic scenario was reconsidered in the full range of inelastic mass splitting allowed by kinematics. The authors argued that the direct detection experiments employing targets with heavy nuclei could be used to explore the so-called "inelastic frontier" - in which the dark matter mass splitting is in several hundred $\mathrm{keV} / \mathrm{c}^{2}$ range. They hypothesized that the four events with high nuclear recoil energy identified by the CRESST experiment [10] might be due to the WIMP inelastic scattering.

\footnotetext{
* Corresponding author: chenxun@sjtu.edu.cn

$\dagger$ Corresponding author: abdusalam291@qq.com

¥ Spokesperson: xdji@sjtu.edu.cn
}

Following the suggestion in Ref. [9], in this article, we report a search of the WIMP inelastic scattering using the data from the first physics run of the PandaX-II experiment from March 9 to June 30, 2016 (Run 9, 79.6 live days).

The PandaX-II detector has been described in detail in Ref. [11]. The central component is a dualphase xenon time-projection-chamber (TPC) with a dodecagonal cross section confined by polytetrafluoroethylene (PTFE) walls, with a sensitive xenon target mass of $580 \mathrm{~kg}$. The primary scintillation photons $(S 1)$ and the secondary proportional scintillation $(S 2)$ are detected by two arrays of Hamamatsu R11410-20 photomultipliers (PMTs) located at the top and bottom. In comparison to the elastic WIMP scattering, due to the mass splitting $\delta$, only WIMPs with sufficiently large velocity (or kinetic energy) relative to the nucleus could be scattered inelastically, resulting in a reduced phase space. A non-zero minimal recoil energy $E_{\mathrm{R}}^{\min }$ also arises from the requirement on the minimum kinetic energy.

Suppression of the kinetic phase space in inelastic WIMP-nucleus scattering can be described by the minimal required relative velocity $v_{\min }$ for a given recoil energy $E_{\mathrm{R}}$. It is

$$
v_{\min }=\frac{1}{\sqrt{2 E_{\mathrm{R}} m_{\mathrm{N}}}}\left(\frac{E_{\mathrm{R}} m_{\mathrm{N}}}{\mu}+\delta\right),
$$


where $m_{\mathrm{N}}$ is the mass of target nucleus and $\mu$ is the reduced mass of the system. The global minimal velocity is determined only by the mass splitting and the reduced mass,

$$
v_{\min }^{\text {global }}=\sqrt{\frac{2 \delta}{\mu}} .
$$

Therefore, a more massive WIMP will lead to larger $\mu$ and smaller $v_{\min }^{\text {global }}$, resulting in a lesser suppression of the phase space. Since most of the nuclei used for dark matter detection have a mass on the order of $100 \mathrm{GeV} / \mathrm{c}^{2}$, when WIMP mass is larger than $10 \mathrm{TeV} / \mathrm{c}^{2}, \mu$ approaches $m_{\mathrm{N}}$ and $v_{\mathrm{min}}^{\text {global }}$ approaches a constant. Therefore, we selected $1 \mathrm{TeV} / \mathrm{c}^{2}$ and $10 \mathrm{TeV} / \mathrm{c}^{2}$ as two reference dark matter masses to perform the analysis, following the detailed discussion in Ref. [9].

The differential energy spectrum of inelastic scattering is given by

$$
\frac{\mathrm{d} R}{\mathrm{~d} E}=\frac{\rho_{0}}{m_{\chi} m_{\mathrm{N}}} \int_{v_{\min }}^{v_{\max }} \frac{\mathrm{d} \sigma}{\mathrm{d} E} v f(\vec{v}, t) \mathrm{d}^{3} v,
$$

where $\rho_{0}$ is the local density of WIMPs, $m_{\chi}$ is the WIMP mass, $\mathrm{d} \sigma / \mathrm{d} E$ is the differential scattering cross section, and $f(\vec{v}, t)$ is the WIMP velocity distribution in the earth's rest frame. The formula has the same form as that of the elastic scattering, with the only difference coming from the lower limit $v_{\min }$ of the integral of velocity distribution. The upper limit $v_{\max }$ is determined by the escape velocity $v_{\text {esc }}$ of dark matter in the Galaxy. We consider the DM-nuclei scattering to be spin and energy independent, in which the differential cross section $\mathrm{d} \sigma / \mathrm{d} E$ takes the form of

$$
\frac{\mathrm{d} \sigma}{\mathrm{d} E}=\frac{m_{\mathrm{N}} \sigma_{n}}{2 \mu_{n}^{2} v^{2}} \cdot\left(Z \cdot f^{p}+(A-Z) \cdot f^{n}\right)^{2} F_{\mathrm{SI}}^{2}(E) .
$$

The parameter $\mu_{n}$ is the reduced mass of the WIMPnucleon system, and $\sigma_{n}$ is the spin independent (SI) WIMP-nucleon cross section at zero momentum transfer in the elastic $(\delta=0)$ limit. $f^{n, p}$ are the effective WIMP coupling to neutron and proton, respectively. $Z$ and $A$ are the atomic and mass number of the target nucleus. $F_{\mathrm{SI}}^{2}(E)$ is the SI nuclear form factor, and we use the Helm parameterization [12] in our analysis[13]. Three WIMP parameters are required to calculate the spectrum. $m_{\chi}, \sigma_{n}$ are explicit in Eqn. 4, and the third one, $\delta$, determines the lower limit $v_{\min }$ of the integral. With the standard assumptions $\rho_{0}=0.3 \mathrm{GeV} / \mathrm{c}^{2} / \mathrm{cm}^{3}$, $v_{\text {esc }}=544 \mathrm{~km} / \mathrm{s}$, the solar system average circular velocity $220 \mathrm{~km} / \mathrm{s}$, and the average earth velocity $232 \mathrm{~km} / \mathrm{s}$, we calculate the expected event rate for inelastic scattering by taking the SI WIMP-nucleon cross section $\sigma_{n}$ to be $10^{-40} \mathrm{~cm}^{2}$ at the dark matter mass of $1 \mathrm{TeV} / \mathrm{c}^{2}$ and $10 \mathrm{TeV} / \mathrm{c}^{2}$, respectively, with four different mass splitting values. The results are shown in Fig. 1. The minimal recoil energy is below $40 \mathrm{keV}$ for both dark matter masses when $\delta=200 \mathrm{keV} / \mathrm{c}^{2}$, so it could be accessed with the

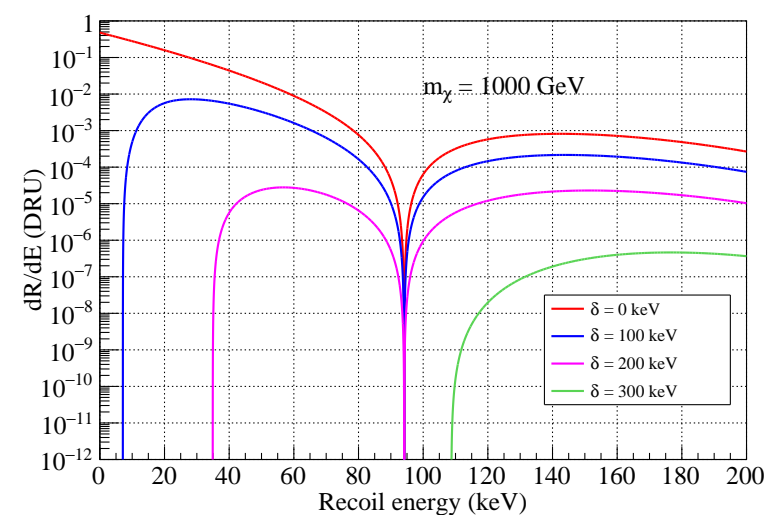

(a) WIMP mass $m_{\chi}=1 \mathrm{TeV} / \mathrm{c}^{2}$.

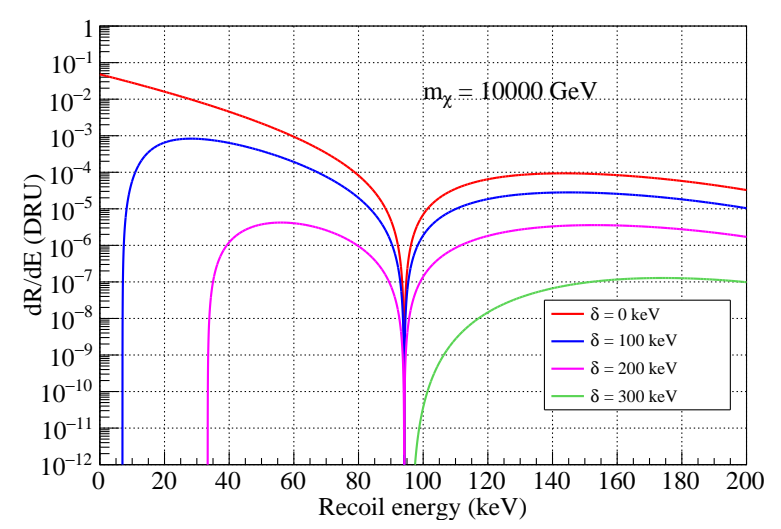

(b) WIMP mass $m_{\chi}=10 \mathrm{TeV} / \mathrm{c}^{2}$.

FIG. 1: The expected event rate for scattering between WIMPs and Xe nuclei at the WIMP masses of 1 $\mathrm{TeV} / \mathrm{c}^{2}$ (top) and $10 \mathrm{TeV} / \mathrm{c}^{2}$ (bottom), respectively, and the mass splitting values of $0,100,200,300 \mathrm{keV} / \mathrm{c}^{2}$. The SI WIMP-nucleon cross section $\sigma_{n}$ is fixed at $10^{-40}$ $\mathrm{cm}^{2}$. The rate is given in the unit of $\mathrm{keV}^{-1} \mathrm{~kg}^{-1} \mathrm{day}^{-1}$

(DRU).

previously published PandaX-II data [14]. But for the case that $\delta=300 \mathrm{keV} / \mathrm{c}^{2}$, the minimal recoil energy is larger than $100 \mathrm{keV} / \mathrm{c}^{2}$ for $m_{\chi}=1 \mathrm{TeV} / \mathrm{c}^{2}$, therefore falling out of the energy range of the same data selection.

In PandaX-II, the data selection is made by requiring $S 1$ and $S 2$ be within a certain range (acceptance). In combination with other data selection cuts, these correspond to an overall detection efficiency curve as a function of true nuclear recoil energy. In the analysis of SI elastic WIMP-nucleon scattering with PandaX-II data [14], the reported average energy window was between 4.6 to $35.0 \mathrm{keV}_{n r}$ (nuclear recoil energy). However, due to the smearing of $S 1$ and $S 2$, nuclear recoil events with energy up to $100 \mathrm{keV}_{n r}$ could still enter into the search window, as indicated in Fig. 2.

The expected signal distributions of $\log _{10}(S 2 / S 1)$ ver- 


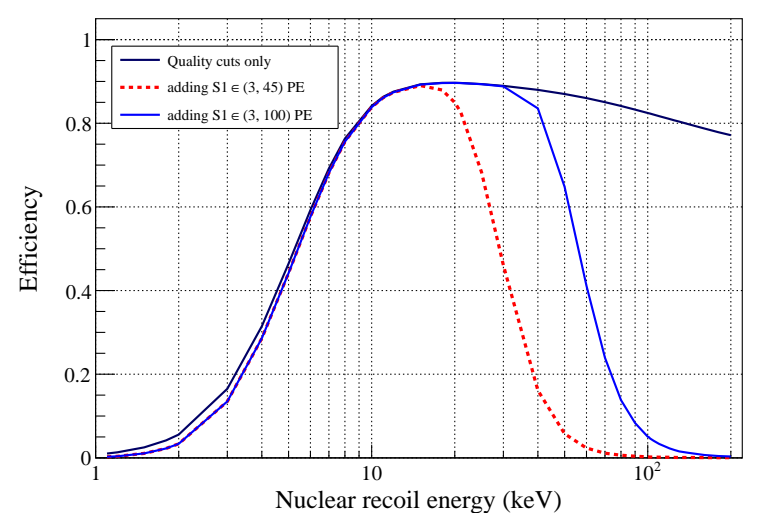

FIG. 2: The detection efficiency of PandaX-II experiment as a function of the nuclear recoil energy. The dark blue curve represents the selection efficency of data quality cuts (see Ref. [14]). The red dashed line is obtained with additional $S 1$ range cut of $(3,45) \mathrm{PE}$ and an upper range of 10,000 PE for $S 2$. The blue solid line is obtained with an extended $S 1$ and $S 2$ window: $S 1$ within $(3,100) \mathrm{PE}$, and $\mathrm{S} 2$ less than 12,000 PE.

sus $S 1$ for $1 \mathrm{TeV} / \mathrm{c}^{2}$ WIMP with different values of $\delta$ in PandaX-II are shown in Fig. 3. Besides the parameters used to calculate the event rate, detector parameters including the photon detection efficiency (PDE), electron extraction efficiency (EEE), single electron gain (SEG), and the electron lifetime, are used to generate the distributions. To simulate $S 1$ and $S 2$ given a nuclear recoil energy, the NEST model [15] was used. At a given value of $\sigma_{n}$, with the increasing of mass splitting, the expected number of event decreases, and the signal band moves towards higher energy. But a sizable fraction of events can still be found with $S 1<100 \mathrm{PE}$ even when $\delta=300$ $\mathrm{keV} / \mathrm{c}^{2}$.

We followed the identical data selection procedure reported in Ref. [14]. All data quality cuts remained the same. After the same fiducial volume cut, the liquid xenon mass was estimated to be $329 \pm 16 \mathrm{~kg}$. The total exposure in Run 9 is about $2.6 \times 10^{4} \mathrm{~kg}$-day. The final candidate selection was made by expanding the $S 1$ upper window to $100 \mathrm{PE}$ and $S 2$ (corrected) upper window to $12,000 \mathrm{PE}$, corresponding to an average upper energy of $18.3 \mathrm{keV}_{e e}$ or $68.6 \mathrm{keV}_{n r}$. Though a higher nuclear recoil energy window has been studied by XENON100 [16], we conservatively choose the value of $100 \mathrm{PE}$ with two considerations. Firstly, the ER background in higher energy region is greatly enhanced by the $33 \mathrm{keV}$ K-shell $\mathrm{x}$ ray generated in the ${ }^{127} \mathrm{Xe}$ electron capture process. Secondly, our ER background model is only optimized in the region with energy up to the end-point decay energy of tritium, i.e., $18.6 \mathrm{keV}_{e e}$. This improves the detection efficiency for events with higher nuclear recoil energies, as shown in Fig. 2. In total, 716 events are observed with the expanded signal window. Based on the same back-
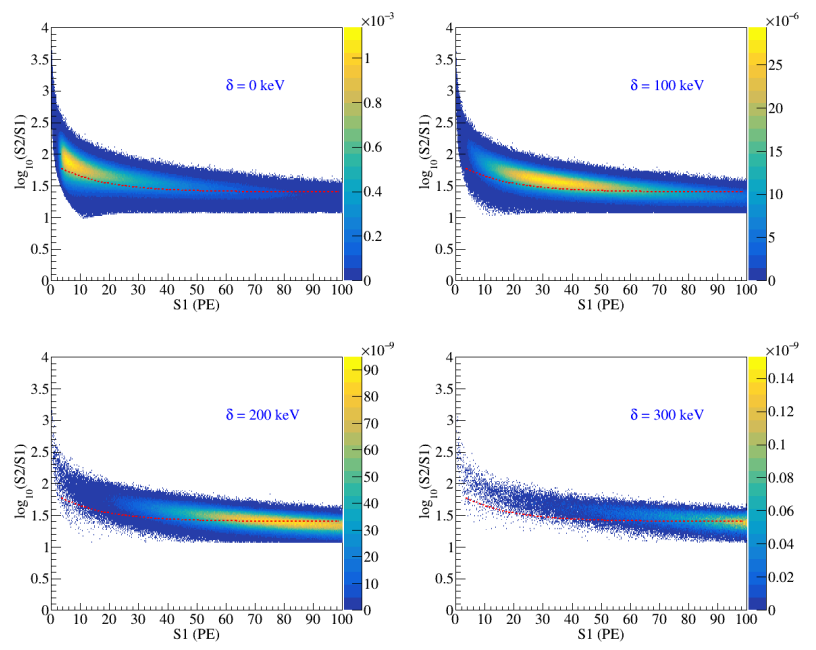

FIG. 3: The expected signal distribution of WIMP-nucleon scattering at the WIMP mass of $m_{\chi}=1$ $\mathrm{TeV} / \mathrm{c}^{2}$ and the mass splitting $\delta$ values of $0,100,200$ and $300 \mathrm{keV} / \mathrm{c}^{2}$, with one set of PandaX-II detector condition parameters. The SI WIMP-nucleon cross section $\sigma_{n}$ is fixed at $10^{-40} \mathrm{~cm}^{2}$. The total expected event rates at each mass splitting are 5.16, 0.21, $5.23 \times 10^{-4}$ and $3.18 \times 10^{-7} \mathrm{evt} / \mathrm{kg} /$ day, respectively.

The $35 \%$ quantile of the NR band from AmBe calibration (red dashed line) is overlaid in each plot.

\begin{tabular}{cccc|c|c}
\hline \hline & ER & Accidental Neutron & $\begin{array}{c}\text { Total } \\
\text { Expected }\end{array}$ & $\begin{array}{c}\text { Total } \\
\text { Observed }\end{array}$ \\
\hline Run 9 & 729.4 & 14.2 & 1.1 & $744 \pm 88$ & 716 \\
\hline $\begin{array}{c}\text { Below 35\% } \\
\text { quantile of } \\
\text { NR band }\end{array}$ & 2.52 & 0.73 & 0.42 & $3.7 \pm 0.4$ & 4 \\
\hline
\end{tabular}

TABLE I: The expected background events in Run 9 with expanded signal window. The fractional uncertainties of expected events are the same as those in Ref. [14].

ground model as in Ref. [14], the expected total number of background events is $744 \pm 88$. The expected background composition can be found in Table I.

The spatial distribution of events before and after the FV cut with the extended signal range is shown in Fig. 4. The events show good uniformity within the FV. The $\log _{10}(S 2 / S 1)$ vs. $S 1$ distribution of these events is shown in Fig. 5. The reference line is chosen to be the $35 \%$ quantile of the calibration AmBe NR band reported in Ref. [14]. Four events are identified below the line. They are also marked in Fig. 4. The event with the smallest $S 1$ value was also reported in Ref. [14]. The event with a $S 1$ value of $51.8 \mathrm{PE}$ appeared at the top edge of the FV. After further investigation of its waveform, we found that this event contains spurious signals which could be produced by the discharge on the electrodes. The waveforms 


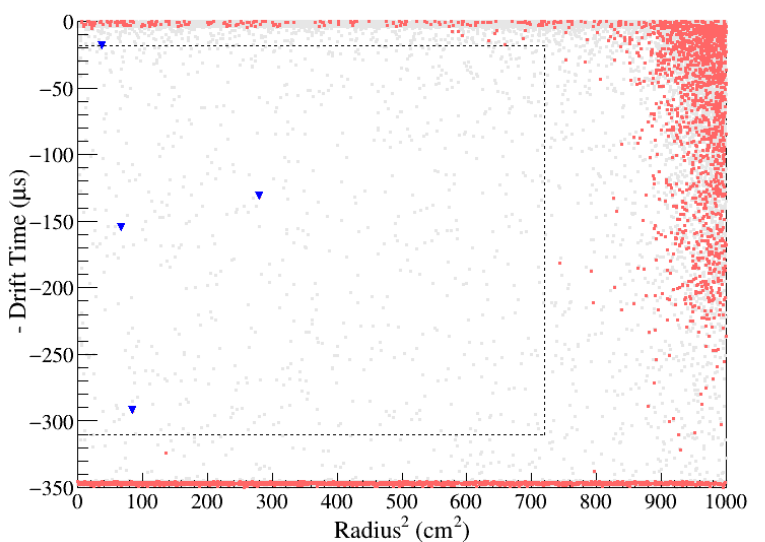

FIG. 4: Spatial distribution of events before the FV cut with expanded signal window (gray points), and those of events below the reference acceptance line (outside FV: red points; inside FV: blue triangles). The FV cuts are indicated by the black dashed lines.

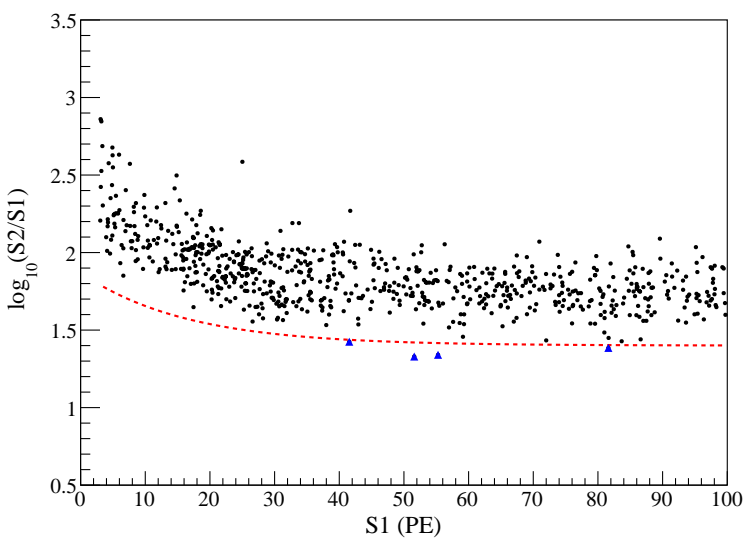

FIG. 5: The $\log _{10}(S 2 / S 1)$ vs. $S 1$ distribution of selected events within the expanded signal window. A reference acceptance line is indicated as the red dashed

line, below which four events (blue triangles) are identified.

of the other two events are of good quality. The event with a $S 1$ value of 55.0 PE has a larger distance from the reference line, but it is located close to the bottom of the FV.

We used all final 716 candidates to derive the exclusion limits for the scattering cross section between WIMPs and nucleons at the two reference dark matter masses of 1 $\mathrm{TeV} / \mathrm{c}^{2}$ and $10 \mathrm{TeV} / \mathrm{c}^{2}$. The profile likelihood fitting was applied to the distribution of $S 1$ and $S 2$. We used identical background models from Ref. [14]. The final $90 \%$ confidence level (C.L.) cross section upper limits were calculated with the CLs approach [17, 18], and are shown in Fig. 6. In both cases, our limit curves lie between the 1- $\sigma$ and $2-\sigma$ sensitivity bands when the mass splitting is within the $(100,200) \mathrm{keV} / \mathrm{c}^{2}$ range, as a result of three observed events below the reference acceptance line. The limit calculated with data in the original signal window ( $S 1$ between 3-45 PE), also shown in the figure, is tighter at lower mass splitting, since only one event was found below the reference line. To study the impact of nuclear recoil model to the derived limit, we also plot the limits calculated based on the "best-tuned" NEST from LUX D-D calibration [19] for a WIMP-mass of $1 \mathrm{TeV} / \mathrm{c}^{2}$. Due to reduction of the NR acceptance, the alternative limit is weaker but not too far beyond the $+1 \sigma$ sensitivity band. In comparison to the earlier phenomenological treatment (up to about $200 \mathrm{keV} / \mathrm{c}^{2}$ for LUX-PandaX-II) in Ref. [9], this analysis extends to a wider mass splitting range up to $300 \mathrm{keV} / \mathrm{c}^{2}$. Our results are also incompatible with the hypothesis that the four high nuclear recoil events observed by CRESST [10] were due to inelastic WIMPs with a mass of $1 \mathrm{TeV} / \mathrm{c}^{2}$ and mass splitting around 200 $\mathrm{keV} / \mathrm{c}^{2}$ (which would suggest a cross section at $\mathcal{O}\left(10^{-39}\right)$ $\left.\mathrm{cm}^{2}\right)$.

In conclusion, we report the inelastic WIMP search results using PandaX-II Run 9 data with an exposure of $2.6 \times 10^{4} \mathrm{~kg}$-day. We expanded the search signal window and set the $90 \%$ upper limits on the SI WIMP-nucleon cross section for the first time for dark matter masses of $1 \mathrm{TeV} / \mathrm{c}^{2}$ and $10 \mathrm{TeV} / \mathrm{c}^{2}$ with inelastic mass splittings up to $300 \mathrm{keV} / \mathrm{c}^{2}$. The search strategy can be further improved with the continuation of the PandaX-II operation, which will amount to a factor 4 increase in the total exposure.

\section{ACKNOWLEDGMENTS}

This project has been supported by a 985-III grant from Shanghai Jiao Tong University, grants from National Science Foundation of China (Nos. 11365022, 11435008, 11455001, 11505112 and 11525522), a grant from the Ministry of Science and Technology of China (No. 2016YFA0400301). We thank the support of a key laboratory grant from the Office of Science and Technology, Shanghai Municipal Government (No. 11DZ2260700), and the support from the Key Laboratory for Particle Physics, Astrophysics and Cosmology, Ministry of Education. This work is supported in part by the Chinese Academy of Sciences Center for Excellence in Particle Physics (CCEPP). We would like to thank Dr. Jian-Hui Zhang for the useful discussions on the integral of WIMP velocity distribution. We also thank Dr. Patrick J. Fox for motivating this work and useful discussion on the manuscript. Finally, we thank the following organizations for indispensable logistics and other supports: the CJPL administration and the Yalong River Hydropower Development Company Ltd. 


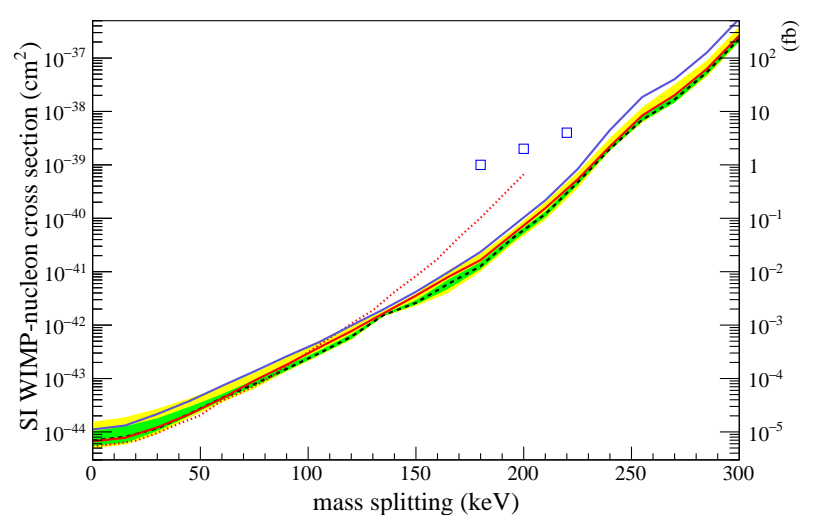

(a) WIMP mass $m_{\chi}=1 \mathrm{TeV} / \mathrm{c}^{2}$.

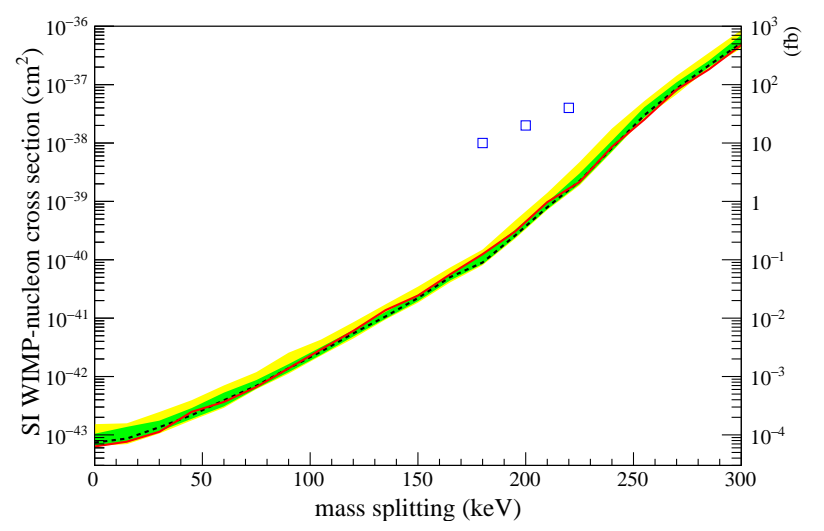

(b) WIMP mass $m_{\chi}=10 \mathrm{TeV} / \mathrm{c}^{2}$.

FIG. 6: The 90\% C.L. upper limits (red solid line) for the SI WIMP-nucleon cross section $\sigma_{n}$ from the

PandaX-II run 9 data with expanded signal window at two reference dark matter masses of $1 \mathrm{TeV} / \mathrm{c}^{2}$ (top) and

$10 \mathrm{TeV} / \mathrm{c}^{2}$ (bottom). The 1 and $2-\sigma$ sensitivity bands are shown in green and yellow, respectively, and the medians of the sensitivity band are given in black dashed line. The red dotted line gives the upper limits calculated with the original data selection window.

Upper limits calculated with a tuned NEST model by LUX are indicated by the blue solid line. The blue squares indicate the possible WIMP mass splittings and nucleon scattering cross sections by the CRESST data [9].

[1] T. Marrodn Undagoitia and L. Rauch, J. Phys. G43, 013001 (2016), arXiv:1509.08767 [physics.ins-det].

[2] J. Liu, X. Chen, and X. Ji, Nature Phys. 13, 212 (2017).

[3] D. Tucker-Smith and N. Weiner, Phys. Rev. D64, 043502 (2001), arXiv:hep-ph/0101138 [hep-ph].

[4] D. Tucker-Smith and N. Weiner, Phys. Rev. D72, 063509 (2005), arXiv:hep-ph/0402065 [hep-ph].

[5] S. Chang, G. D. Kribs, D. Tucker-Smith, and N. Weiner,
Phys. Rev. D79, 043513 (2009), arXiv:0807.2250 [hep$\mathrm{ph}$.

[6] R. Bernabei et al., Eur. Phys. J. C73, 2648 (2013), arXiv:1308.5109 [astro-ph.GA].

[7] K. Abe et al. (XMASS), Phys. Lett. B759, 272 (2016), arXiv:1511.04807 [astro-ph.CO].

[8] E. Aprile et al. (XENON), Phys. Rev. Lett. 118, 101101 (2017), arXiv:1701.00769 [astro-ph.CO]. 
[9] J. Bramante, P. J. Fox, G. D. Kribs, and A. Martin, Phys. Rev. D94, 115026 (2016), arXiv:1608.02662 [hepph].

[10] G. Angloher et al. (CRESST), Eur. Phys. J. C76, 25 (2016), arXiv:1509.01515 [astro-ph.CO].

[11] A. Tan et al. (PandaX), Phys. Rev. D93, 122009 (2016), arXiv:1602.06563 [hep-ex].

[12] J. D. Lewin and P. F. Smith, Astropart. Phys. 6, 87 (1996).

[13] Other nuclear structure input, e.g. Ref. [20], would have little effects for mass splitting less than $100 \mathrm{keV} / \mathrm{c}^{2}$, but bring sizable change for masses above. For example, the integrated rate for delta $=300 \mathrm{keV}$ would reduce by a factor of 2.4 .

[14] A. Tan et al. (PandaX-II), Phys. Rev. Lett. 117, 121303
(2016), arXiv:1607.07400 [hep-ex].

[15] B. Lenardo, K. Kazkaz, M. Szydagis, and M. Tripathi, IEEE Trans. Nucl. Sci. 62, 3387 (2015), arXiv:1412.4417 [astro-ph.IM].

[16] E. Aprile et al. (XENON), (2017), arXiv:1705.02614 [astro-ph.CO].

[17] A. L. Read, J. Phys. G28, 2693 (2002).

[18] T. Junk, Nucl. Instrum. Meth. A434, 435 (1999), arXiv:9902006 [hep-ex].

[19] D. S. Akerib et al. (LUX), (2016), arXiv:1608.05381 [physics.ins-det].

[20] L. Vietze, P. Klos, J. Menndez, W. C. Haxton, and A. Schwenk, Phys. Rev. D91, 043520 (2015), arXiv:1412.6091 [nucl-th]. 